\title{
Multivariable integrated evaluation of model performance with the vector field evaluation diagram
}

\author{
Zhongfeng $\mathrm{Xu}^{1}$, Ying $\mathrm{Han}^{1}$, and Congbin $\mathrm{Fu}^{2,1}$ \\ ${ }^{1}$ CAS Key Laboratory of Regional Climate-Environment for Temperate East Asia, Institute of Atmospheric Physics, \\ Chinese Academy of Sciences, Beijing, China \\ ${ }^{2}$ Institute for Climate and Global Change Research and School of Atmospheric Sciences, Nanjing University, Nanjing, China \\ Correspondence to: Zhongfeng Xu (xuzhf@tea.ac.cn)
}

Received: 8 April 2017 - Discussion started: 12 May 2017

Revised: 14 September 2017 - Accepted: 14 September 2017 - Published: 23 October 2017

\begin{abstract}
This paper develops a multivariable integrated evaluation (MVIE) method to measure the overall performance of climate model in simulating multiple fields. The general idea of MVIE is to group various scalar fields into a vector field and compare the constructed vector field against the observed one using the vector field evaluation (VFE) diagram. The VFE diagram was devised based on the cosine relationship between three statistical quantities: root mean square length (RMSL) of a vector field, vector field similarity coefficient, and root mean square vector deviation (RMSVD). The three statistical quantities can reasonably represent the corresponding statistics between two multidimensional vector fields. Therefore, one can summarize the three statistics of multiple scalar fields using the VFE diagram and facilitate the intercomparison of model performance. The VFE diagram can illustrate how much the overall root mean square deviation of various fields is attributable to the differences in the root mean square value and how much is due to the poor pattern similarity. The MVIE method can be flexibly applied to full fields (including both the mean and anomaly) or anomaly fields depending on the application. We also propose a multivariable integrated evaluation index (MIEI) which takes the amplitude and pattern similarity of multiple scalar fields into account. The MIEI is expected to provide a more accurate evaluation of model performance in simulating multiple fields. The MIEI, VFE diagram, and commonly used statistical metrics for individual variables constitute a hierarchical evaluation methodology, which can provide a more comprehensive evaluation of model performance.
\end{abstract}

\section{Introduction}

Climate models play a very crucial role in a variety of climate-related studies including, e.g., climate dynamics, the detection and attribution of climate change, the projection of future climates and environments, and adaptation to future climate change (IPCC, 2012, 2013). All these studies strongly rely on the performance of climate models. Model evaluation and intercomparison have become increasingly important, especially because a number of climate models are available at present. A total of 29 modelling groups and 60 climate models are involved in the Coupled Model Intercomparison Project Phase 5 (CMIP5) and more are expected to be included in its next phase (Eyring et al., 2016). In addition, more and more regional climate models have been used in regional model downscaling and intercomparison projects (e.g., Fu et al., 2005; van der Linden and Mitchell, 2009; Mearns et al., 2009; Giorgi and Gutowski, 2015). Thus, how to concisely summarize and evaluate model performance is extremely important for climate model intercomparison, development, and application.

The Taylor diagram provides a very efficient way to summarize multiple aspects of model performance in simulating scalar fields (Taylor, 2001). Gleckler et al. (2008) introduced a suite of metrics, e.g., decomposed mean square error, and relative error metrics, which were used to characterize the model performance for various applications. Xu et al. (2016) devised a vector field evaluation (VFE) diagram, which can be regarded as a generalized Taylor diagram, to evaluate the model performance in simulating vector fields, such as vector winds and temperature gradients. Most metrics, e.g., root mean square error, correlation coefficient, and standard devi- 
ation, measure the model performance in simulating an individual variable (Gleckler et al., 2008). It is a common view that no model performs better than others in every aspect. For example, among various models, one model can show the best performance in simulating air temperature but may have a poor performance in simulating precipitation. In this case, how can researchers select the best model if both temperature and precipitation are of great concern in a study? A popular approach is to show the relative errors of various variables from different models using a portrait diagram (e.g., Gleckler et al., 2008; Pincus et al., 2008). The portrait diagram illustrates model errors for each individual variable and can provide an overview of the model performance in simulating various variables. However, the portrait diagram cannot give a quantitative evaluation of the overall performance of climate models in simulating multiple fields. To measure the overall model performance, Gleckler et al. (2008) proposed an exploratory index, termed the model climate performance index (MCPI), by averaging each model's relative errors across multiple fields. Note that the MCPI only considers the root mean square errors (RMSEs) of various fields. The RMSE can be interpreted as a function of the correlation coefficient and standard deviation (Murphy, 1988; Taylor, 2001; Pincus et al., 2008; Pierce et al., 2009). Therefore, the RMSE takes both the correlation coefficient and standard deviation into account. However, the RMSE cannot explicitly measure the correlation coefficient and standard deviation. For example, the same RMSE can correspond to very different correlation coefficients and standard deviations, especially for large RMSE values.

In this paper, we propose a more comprehensive multivariable integrated evaluation (MVIE) method, which can summarize multiple statistics of model performance in terms of multiple variables, for climate model evaluation. The general idea is to group $M$ scalar fields into an $M$-dimensional vector field with each dimension representing a scalar field. Such a constructed vector field integrates multiple variables and can be assessed using the VFE diagram. The VFE diagram can concisely summarize the degree of correspondence between simulated and observed vector fields in terms of multiple statistics (Xu et al., 2016). Therefore, the VFE diagram can be a powerful tool for the MVIE of model performance. To achieve the goal of MVIE, in Sect. 2, we generalize the VFE diagram to evaluate $M$-dimensional vector fields and interpret three statistical quantities in the VFE diagram from the viewpoint of MVIE. Section 3 presents the approach of MVIE with the VFE diagram. A summary and discussion are provided in Sect. 4.

\section{Constructing the VFE diagram for multidimensional vector fields}

$\mathrm{Xu}$ et al. (2016) constructed the VFE diagram in terms of two-dimensional vector fields. There are three statistical quantities in the VFE diagram, i.e., root mean square length (RMSL) of a vector field, vector similarity coefficient (VSC), and root mean square vector deviation (RMSVD) between two vector fields. In this section, each quantity will be defined and interpreted from the viewpoint of MVIE. Thereafter, we will construct the VFE diagram for multidimensional vector fields.

\subsection{Root mean square length of a vector field}

Consider two vector fields $\boldsymbol{A}$ and $\boldsymbol{B}$, which can be spatial and/or temporal fields. Assume that vector fields $\boldsymbol{A}$ and $\boldsymbol{B}$ are derived from a climate model simulation and observation, respectively. Without loss of generality, vector fields $\boldsymbol{A}$ and $\boldsymbol{B}$ can be written as a pair of vector sequences:

$\begin{array}{ll}\boldsymbol{A}_{j}=\left(a_{1 j}, a_{2 j}, \ldots, a_{M j}\right) ; & j=1,2, \ldots, N \\ \boldsymbol{B}_{j}=\left(b_{1 j}, b_{2 j}, \ldots, b_{M j}\right) ; & j=1,2, \ldots, N .\end{array}$

Each vector field, e.g., $\boldsymbol{A}$, consists of $N$ discrete vectors (in time and/or space). Each vector, e.g., $\boldsymbol{A}_{j}$, in $M$-dimensional Euclidean space is identified with the tuples of $M$ real numbers $\left(a_{1 j}, a_{2 j}, \ldots, a_{M j}\right)$. Each real number represents the position of the perpendicular projection of the vector onto individual axes of an $M$-dimensional Cartesian coordinate system. The norms of vectors $\boldsymbol{A}_{j}$ and $\boldsymbol{B}_{j}$, the intuitive notion of length, are written as

$$
\begin{aligned}
\left\|\boldsymbol{A}_{j}\right\| & =\left(\sum_{i=1}^{M} a_{i j}^{2}\right)^{\frac{1}{2}} \\
\left\|\boldsymbol{B}_{j}\right\| & =\left(\sum_{i=1}^{M} b_{i j}^{2}\right)^{\frac{1}{2}} .
\end{aligned}
$$

The root mean square lengths (RMSLs) for vector fields $\boldsymbol{A}$ and $\boldsymbol{B}$ are, respectively, defined as

$$
L_{A}=\sqrt{\frac{1}{N} \sum_{j=1}^{N}\left\|\boldsymbol{A}_{j}\right\|^{2}}
$$

and

$$
L_{B}=\sqrt{\frac{1}{N} \sum_{j=1}^{N}\left\|\boldsymbol{B}_{j}\right\|^{2}} .
$$

The square of $L_{A}$ is written as

$$
\begin{aligned}
L_{A}^{2} & =\frac{1}{N} \sum_{j=1}^{N}\left\|\boldsymbol{A}_{j}\right\|^{2} \\
& =\frac{1}{N} \sum_{j=1}^{N} \sum_{i=1}^{M} a_{i j}^{2} \\
& =\sum_{i=1}^{M}\left(\frac{1}{N} \sum_{j=1}^{N} a_{i j}^{2}\right)
\end{aligned}
$$




$$
=\sum_{i=1}^{M} L_{a i}^{2}
$$

Similarly, we have

$$
\begin{aligned}
L_{B}^{2} & =\frac{1}{N} \sum_{j=1}^{N}\left\|\boldsymbol{B}_{j}\right\|^{2} \\
& =\frac{1}{N} \sum_{j=1}^{N} \sum_{i=1}^{M} b_{i j}^{2} \\
& =\sum_{i=1}^{M}\left(\frac{1}{N} \sum_{j=1}^{N} b_{i j}^{2}\right) \\
& =\sum_{i=1}^{M} L_{b i}^{2},
\end{aligned}
$$

where

$$
L_{a i}=\sqrt{\frac{1}{N} \sum_{j=1}^{N} a_{i j}^{2}}
$$

and

$$
L_{b i}=\sqrt{\frac{1}{N} \sum_{j=1}^{N} b_{i j}^{2}}
$$

are the rms values of the $i$ th component of the vector fields $\boldsymbol{A}$ and $\boldsymbol{B}$, respectively. The RMSL of vector field $\boldsymbol{A}$ reflects the total rms value across all components of the vector field (Eq. 3). If we break down each variable into its mean and anomaly, it is easy to prove that the mean square value equals the square of the mean plus variance (Eq. A4). If the vector field is grouped with various scalar fields, the RMSL represents the overall mean value and variance of all scalar fields.

\subsection{Vector similarity coefficient between two vector fields}

In the same way as for the vector similarity coefficient (VSC) for two-dimensional vector fields (Xu et al., 2016), the VSC for $M$-dimensional vector fields can be defined as

$$
R_{\mathrm{v}}=\frac{\sum_{j=1}^{N} \boldsymbol{A}_{j} \cdot \boldsymbol{B}_{j}}{\sqrt{\sum_{j=1}^{N}\left\|\boldsymbol{A}_{j}\right\|^{2}} \sqrt{\sum_{j=1}^{N}\left\|\boldsymbol{B}_{j}\right\|^{2}}} .
$$

The normalized vectors are written as

$$
\begin{aligned}
\boldsymbol{A}_{j}^{*} & =\frac{\boldsymbol{A}_{j}}{L_{A}}=\left(a_{1 j}^{*}, a_{2 j}^{*}, \ldots, a_{M j}^{*}\right) ; \quad j=1,2, \ldots, N \\
\boldsymbol{B}_{j}^{*} & =\frac{\boldsymbol{B}_{j}}{L_{B}}=\left(b_{1 j}^{*}, b_{2 j}^{*}, \ldots, b_{M j}^{*}\right) ; \quad j=1,2, \ldots, N .
\end{aligned}
$$

With the aid of Eqs. (1) and (2), we have

$$
\sum_{j=1}^{N}\left\|\boldsymbol{A}_{j}^{*}\right\|^{2}=\sum_{j=1}^{N}\left\|\boldsymbol{B}_{j}^{*}\right\|^{2}=N .
$$

We can also represent Eq. (7) in the following form:

$$
\begin{aligned}
R_{\mathrm{V}} & =\frac{1}{N} \sum_{j=1}^{N} \boldsymbol{A}_{j}^{*} \cdot \boldsymbol{B}_{j}^{*} \\
& =\frac{1}{N} \sum_{j=1}^{N} \sum_{i=1}^{M} a_{i j}^{*} b_{i j}^{*} .
\end{aligned}
$$

VSC can be interpreted as the mean of inner products between normalized and paired vectors $\boldsymbol{A}_{j}^{*}$ and $\boldsymbol{B}_{j}^{*}$. The squared Euclidean distance (SED) between $\boldsymbol{A}_{j}^{*}$ and $\boldsymbol{B}_{j}^{*}$ is defined as follows:

$\left\|\boldsymbol{C}_{j}^{*}\right\|^{2}=\left\|\boldsymbol{A}_{j}^{*}-\boldsymbol{B}_{j}^{*}\right\|^{2}$.

With the aid of Eqs. (9) and (10), the sum of all SEDs can be written as

$$
\begin{aligned}
\sum_{j=1}^{N}\left\|\boldsymbol{C}_{j}^{*}\right\|^{2} & =\sum_{j=1}^{N}\left\|\boldsymbol{A}_{j}^{*}-\boldsymbol{B}_{j}^{*}\right\|^{2} \\
& =\sum_{j=1}^{N} \sum_{i=1}^{M}\left(a_{i j}^{*}-b_{i j}^{*}\right)^{2} \\
& =\sum_{j=1}^{N}\left(\sum_{i=1}^{M} a_{i j}^{* 2}+\sum_{i=1}^{M} b_{i j}^{*}-2 \sum_{i=1}^{M} a_{i j}^{*} b_{i j}^{*}\right) \\
& =\sum_{j=1}^{N}\left\|\boldsymbol{A}_{j}^{*}\right\|^{2}+\sum_{j=1}^{N}\left\|\boldsymbol{B}_{j}^{*}\right\|^{2}-2 N \cdot R_{\mathrm{v}} .
\end{aligned}
$$

With the aid of Eq. (8), we obtain

$R_{\mathrm{V}}=1-\frac{1}{2 N} \sum_{j=1}^{N}\left\|\boldsymbol{C}_{j}^{*}\right\|^{2}$.

Given the triangle inequality, $0 \leq\left\|C_{j}^{*}\right\| \leq\left\|\boldsymbol{A}_{j}^{*}\right\|+\left\|\boldsymbol{B}_{j}^{*}\right\|$, we have

$0 \leq\left\|\boldsymbol{C}_{j}^{*}\right\|^{2} \leq\left(\left\|\boldsymbol{A}_{j}^{*}\right\|+\left\|\boldsymbol{B}_{j}^{*}\right\|\right)^{2} \leq 2\left\|\boldsymbol{A}_{j}^{*}\right\|^{2}+2\left\|\boldsymbol{B}_{j}^{*}\right\|^{2}$.

Adding all SEDs together yields

$0 \leq \sum_{j=1}^{N}\left\|\boldsymbol{C}_{j}^{*}\right\|^{2} \leq 2 \sum_{j=1}^{N}\left\|\boldsymbol{A}_{j}^{*}\right\|^{2}+2 \sum_{j=1}^{N}\left\|\boldsymbol{B}_{j}^{*}\right\|^{2}=4 N$.

Substituting Eq. (12) into Eq. (11), we obtain $-1 \leq R_{\mathrm{v}} \leq 1$. Thus, the VSC between two $M$-dimensional vector fields varies from -1 to 1 . The VSC reaches its maximum of 1 when each pair of normalized vectors has exactly the same 
length and direction, i.e., $\boldsymbol{A}_{j}^{*}=\boldsymbol{B}_{j}^{*}$ for all $i(1 \leq i \leq N)$. The VSC reaches its minimum value of -1 when each pair of normalized vectors has exactly the same length but points in opposite directions, i.e., $\boldsymbol{A}_{j}^{*}=-\boldsymbol{B}_{j}^{*}$ for all $i(1 \leq i \leq N)$.

With the aid of Eqs. (1) and (2), Eq. (7) can be written as

$$
\begin{aligned}
R_{\mathrm{v}} & =\frac{1}{N L_{A} L_{B}} \sum_{j=1}^{N} \boldsymbol{A}_{j} \cdot \boldsymbol{B}_{j} \\
& =\frac{1}{N L_{A} L_{B}} \sum_{j=1}^{N} \sum_{i=1}^{M} a_{i j} b_{i j} \\
& =\frac{1}{N L_{A} L_{B}} \sum_{j=1}^{N} \sum_{i=1}^{M} \frac{a_{i j} b_{i j}}{L_{a i} L_{b i}} L_{a i} L_{b i} \\
& =\frac{1}{N L_{A} L_{B}} \sum_{i=1}^{M} \sum_{j=1}^{N} \frac{a_{i j} b_{i j}}{L_{a i} L_{b i}} L_{a i} L_{b i} \\
& =\frac{1}{L_{A} L_{B}} \sum_{i=1}^{M}\left(L_{a i} L_{b i} \frac{1}{N} \sum_{j=1}^{N} \frac{a_{i j} b_{i j}}{L_{a i} L_{b i}}\right) \\
& =\frac{1}{L_{A} L_{B}} \sum_{i=1}^{M} L_{a i} L_{b i} R_{u i},
\end{aligned}
$$

where $L_{a i}$ and $L_{b i}$ are the uncentered rms values of the $i$ th component of vector fields $\boldsymbol{A}$ and $\boldsymbol{B}$ as defined in Eqs. (5) and (6), respectively. $R_{u i}=\frac{\frac{1}{N} \sum_{j=1}^{N} a_{i j} b_{i j}}{L_{a i} L_{b i}}$ is the uncentered pattern correlation coefficient between the $i$ th paired components of vector fields $\boldsymbol{A}$ and $\boldsymbol{B}$. The uncentered pattern correlation coefficient is a variant of Pearson's correlation in which the mean values are not removed. $R_{u i}$ can also be interpreted as the normalized inner product of two $N$-dimensional vectors $\boldsymbol{a}_{i}=\left(a_{i 1}, a_{i 2}, \ldots, a_{i N}\right)$ and $\boldsymbol{b}_{i}=\left(b_{i 1}, b_{i 2}, \ldots, b_{i N}\right)$ :

$$
R_{u i}=\frac{<\boldsymbol{a}_{i} \cdot \boldsymbol{b}_{i}>}{\left\|\boldsymbol{a}_{i}\right\|\left\|\boldsymbol{b}_{i}\right\|}=\frac{\sum_{j=1}^{N} a_{i j} b_{i j}}{\sqrt{\sum_{j=1}^{N} a_{i j}^{2}} \sqrt{\sum_{j=1}^{N} b_{i j}^{2}}} .
$$

The uncentered correlation coefficient can be represented by the cosine of the angle between the $N$-dimensional vectors $\boldsymbol{a}_{i}$ and $\boldsymbol{b}_{i} . R_{u i}$ increases when the arguments of vectors $\boldsymbol{a}_{i}$ and $\boldsymbol{b}_{i}$ approach each other (Eq. 14). Thus, the similarity coefficient between two vector fields $\boldsymbol{A}$ and $\boldsymbol{B}$ can be interpreted as a weighted average of uncentered correlation coefficients across all paired components between two vector fields (Eq. 13).

\subsection{Root mean square vector deviation}

To measure the difference in vector fields $\boldsymbol{A}$ and $\boldsymbol{B}$, a RMSVD is defined as

$$
\begin{aligned}
\operatorname{RMSVD} & =\left[\frac{1}{N} \sum_{j=1}^{N}\left\|\boldsymbol{A}_{j}-\boldsymbol{B}_{j}\right\|^{2}\right]^{\frac{1}{2}} \\
& =\left[\frac{1}{N} \sum_{j=1}^{N} \sum_{i=1}^{M}\left(a_{i j}-b_{i j}\right)^{2}\right]^{\frac{1}{2}} .
\end{aligned}
$$

The square of the RMSVD can be written as

$$
\begin{aligned}
\mathrm{RMSVD}^{2} & =\frac{1}{N} \sum_{j=1}^{N} \sum_{i=1}^{M}\left(a_{i j}-b_{i j}\right)^{2} \\
& =\sum_{i=1}^{M}\left(\frac{1}{N} \sum_{j=1}^{N}\left(a_{i j}-b_{i j}\right)^{2}\right) \\
& =\sum_{i=1}^{M} \operatorname{RMSD}_{i}^{2},
\end{aligned}
$$

where $\operatorname{RMSD}_{i}=\frac{1}{N} \sum_{j=1}^{N}\left(a_{i j}-b_{i j}\right)^{2}$ is the root mean square deviation (RMSD) between the $i$ th paired component of vector fields $\boldsymbol{A}$ and $\boldsymbol{B}$. Thus, the RMSVD measures the overall RMSDs of all components between the original vector fields $\boldsymbol{A}$ and $\boldsymbol{B}$.

\subsection{Construction of VFE diagram for $M$-dimensional vector fields}

With the aid of Eq. (7), the square of the RMSVD can be written as

$$
\begin{aligned}
\mathrm{RMSVD}^{2}= & \frac{1}{N} \sum_{j=1}^{N}\left\|\boldsymbol{A}_{j}-\boldsymbol{B}_{j}\right\|^{2} \\
= & \frac{1}{N} \sum_{j=1}^{N}\left(\left\|\boldsymbol{A}_{j}\right\|^{2}+\left\|\boldsymbol{B}_{j}\right\|^{2}-2 \boldsymbol{A}_{j} \cdot \boldsymbol{B}_{j}\right) \\
= & \frac{1}{N} \sum_{j=1}^{N}\left\|\boldsymbol{A}_{j}\right\|^{2}+\frac{1}{N} \sum_{j=1}^{N}\left\|\boldsymbol{B}_{j}\right\|^{2}-2 R_{\mathrm{V}} \\
& \cdot \sqrt{\frac{1}{N} \sum_{j=1}^{N}\left\|\boldsymbol{A}_{j}\right\|^{2}} \sqrt{\frac{1}{N} \sum_{j=1}^{N}\left\|\boldsymbol{B}_{j}\right\|^{2}} .
\end{aligned}
$$

With the aid of Eqs. (1), (2), and (7), Eq. (17) can be written as

$$
\operatorname{RMSVD}^{2}=L_{A}^{2}+L_{B}^{2}-2 R_{\mathrm{V}} \cdot L_{A} L_{B} .
$$

The RMSVD, $L_{A}, L_{B}$, and $R_{\mathrm{V}}$ are related by the law of cosines (Eq. 18). We can construct the VFE diagram for $M$ dimensional vector fields based on Eq. (18). The VFE diagram and the geometric relationship between $L_{A}, L_{B}, R_{\mathrm{v}}$, 
and the RMSVD are shown in Fig. 1. As for the case of twodimensional vectors ( $\mathrm{Xu}$ et al., 2016), the RMSLs, i.e., $L_{A}$ and $L_{B}$, measure the mean and variance of the lengths of vector fields $\boldsymbol{A}$ and $\boldsymbol{B}$, respectively (Eqs. A4, A5). $R_{\mathrm{v}}$ reflects the pattern similarity between two vector fields. The RMSVD describes the overall difference between two vector fields. Thus, the three statistical quantities can be indicated by a single point on the VFE diagram (Fig. 1).

\section{Multivariable integrated evaluation with the VFE diagram}

\subsection{Methodology}

To evaluate model performance in terms of the simulation of multiple variables, one can group various scalar fields into a vector field and compare the constructed vector field against the observed one using the VFE diagram. For example, we can construct a vector field with temperature and precipitation as its $x$ and $y$ components, respectively. One can certainly use more variables as needed to construct the vector field. Note that the statistical quantities RMSL, VSC, and RMSVD in the VFE diagram are defined in an orthogonal coordinate system in which the axes are perpendicular to each other. There is no requirement for the independence of the variables to be evaluated, e.g., temperature and precipitation which are represented by coordinate values of individual axes. Thus, the VFE diagram can be applied to evaluate any combination of modeled variables against corresponding observational estimates. Given the differences in units and order of magnitude of various variables, we need to normalize all variables before grouping them into a vector field. The normalization can be done by dividing the rms value of each observational estimate as follows:

$$
\begin{aligned}
\boldsymbol{A}_{j}^{\star} & =\left(\frac{a_{1 j}}{L_{b 1}}, \frac{a_{2 j}}{L_{b 2}}, \ldots, \frac{a_{M j}}{L_{b M}}\right)=\left(a_{1 j}^{\star}, a_{2 j}^{\star}, \ldots, a_{M j}^{\star}\right) ; \\
j & =1,2, \ldots, N \\
\boldsymbol{B}_{j}^{\star} & =\left(\frac{b_{1 j}}{L_{b 1}}, \frac{b_{2 j}}{L_{b 2}}, \ldots, \frac{b_{M j}}{L_{b M}}\right)=\left(b_{1 j}^{\star}, b_{2 j}^{\star}, \ldots, b_{M j}^{\star}\right) ; \\
j & =1,2, \ldots, N,
\end{aligned}
$$

where $L_{b i}=\sqrt{\frac{1}{N} \sum_{j=1}^{N} b_{i j}^{2}}$ is the rms value for the $i$ th component of vector field $\boldsymbol{B}$ obtained from observational estimates. Each component of the normalized vector field is dimensionless and on the order of 1 . Thus, the statistics of each component are equally important to the total statistics of the vector fields. The normalization is especially necessary when the variables are of different orders of magnitude. For example, the surface air temperature (SAT) is typically on the order of $10^{0}-10^{1}{ }^{\circ} \mathrm{C}$, but the monthly mean precipitation rate is generally on the order of $10^{-5}-10^{-4} \mathrm{~mm} \mathrm{~s}^{-1}$. Under this circum-

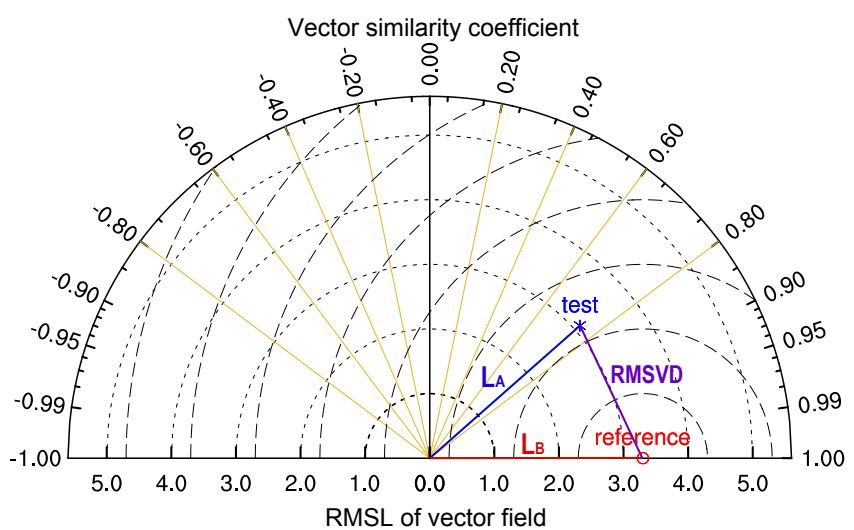

Figure 1. VFE diagram for displaying multiple statistics of two vector fields. The vector similarity coefficient between two vector fields is given by the azimuthal position of the test field. The radial distance from the origin is proportional to the RMSL of the vector field. $L_{A}$ and $L_{B}$ represent the RMSL of the test and reference vector fields, respectively. The RMSVD between the test and reference fields is proportional to their distance (dashed contours are given in the same units as those for the RMSL).

stance, the differences in the RMSL, VSC, and RMSVD between various models would be primarily determined based on the SAT and barely impacted by the precipitation if no normalization was applied. Therefore, in terms of the MVIE of the model performance, the RMSLs, VSC, and RMSVD should be computed using the normalized vector fields $\boldsymbol{A}^{\star}$ and $\boldsymbol{B}^{\star}$. As interpreted in Sect. 2, three statistical quantities in the VFE diagram represent the overall statistics across all components between two vector fields. If the vector fields are grouped by various scalar fields, the VFE diagram can summarize the three statistics of model performance in simulating multiple scalar fields.

\subsection{Application of multivariable integrated evaluation of model performance}

Without loss of generality, we choose the climatological mean SAT and precipitation as well as the temporal standard deviation of the SAT and precipitation as the variables to interpret the MVIE method. Four variables derived from climate models are examined against the corresponding observational estimates. The evaluation is based on the monthly mean datasets derived from the first ensemble run of CMIP5 historical experiments during the period from 1961 to 2000 (Taylor et al., 2012). Three pairs of observed SAT and precipitation datasets are used in this study. The first pair of datasets is the Climatic Research Unit (CRU) gridded SAT and precipitation (Harris, et al., 2014). The second pair of datasets is the University of Delaware air temperature and precipitation (Willmott and Matsuura, 2001). The third pair of datasets is composed of the Global Historical Climatology Network (GHCN) temperature (Fan and van den Dool, 
Table 1. Multiple statistics of CMIP5 models in simulating surface air temperature and precipitation in terms of climatological mean state and interannual variability. Tm (Pm) is the climatological mean surface air temperature (precipitation) in summer (June-July-August). Ta (Pa) is the temporal standard deviation of summer surface air temperature (precipitation). CMIP5 simulations and three individual groups of observational datasets are compared with the ensemble mean of three groups of SAT and precipitation data observed during the period from 1961 to 2000. The rms is the ratio of modeled to observed root mean square values of the spatial pattern for each variable. CORR (RMSD) is the uncentered spatial correlation coefficient (root mean square deviation) between model and observational fields. RMSL, Rv, and RMSVD measure the statistics of two vector fields, which can represent the overall statistics of all fields (Eqs. 3, 13, 16). RMSL was shown as the ratio of model simulated RMSL to the observed RMSL. The rms_std is the standard deviation of four rms values, which describe the dispersion of rms values of Tm, Pm, Ta, and Pa (Eq. 23). MIEI is the multivariable integrated evaluation index (Eq. 24). Model performance is indicated by the color scale; lighter colors denote better model performance.

\begin{tabular}{|c|c|c|c|c|c|c|c|c|c|c|c|c|c|c|c|c|c|}
\hline \multirow{2}{*}{ METRICS } & \multicolumn{4}{|c|}{$\mathrm{rms}$} & \multirow{2}{*}{$\begin{array}{l}\stackrel{D}{\gtrless} \\
\stackrel{\omega}{\Gamma}\end{array}$} & \multicolumn{4}{|c|}{ CORR } & & \multicolumn{4}{|c|}{ RMSD } & \multirow{2}{*}{ 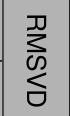 } & \multirow{2}{*}{ 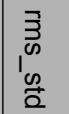 } & \multirow{2}{*}{ 亮 } \\
\hline & $\mathrm{Tm}$ & $\mathrm{Pm}$ & $\mathrm{Ta}$ & $\mathrm{Pa}$ & & $\mathrm{Tm}$ & $\mathrm{Pm}$ & $\mathrm{Ta}$ & $\mathrm{Pa}$ & & $\mathrm{Tm}$ & $\mathrm{Pm}$ & $\mathrm{Ta}$ & $\mathrm{Pa}$ & & & \\
\hline Model 1 & 1.01 & 0.99 & 1.11 & 0.97 & 1.02 & 1.00 & 0.92 & 0.95 & 0.92 & 0.94 & 0.09 & 0.41 & 0.35 & 0.39 & 0.34 & 0.05 & 0.34 \\
\hline Model 2 & 1.04 & 1.01 & 1.13 & 1.05 & 1.06 & 1.00 & 0.83 & 0.93 & 0.84 & 0.90 & 0.10 & 0.58 & 0.42 & 0.58 & 0.46 & 0.04 & 0.44 \\
\hline Model 3 & 1.05 & 0.80 & 1.26 & 0.77 & 0.99 & 1.00 & 0.88 & 0.93 & 0.86 & 0.91 & 0.11 & 0.48 & 0.48 & 0.52 & 0.43 & 0.20 & 0.48 \\
\hline Model 4 & 0.97 & 0.84 & 1.17 & 0.72 & 0.94 & 1.00 & 0.91 & 0.95 & 0.87 & 0.92 & 0.09 & 0.43 & 0.38 & 0.52 & 0.39 & 0.17 & 0.44 \\
\hline Model 5 & 0.99 & 0.94 & 1.43 & 1.19 & 1.15 & 1.00 & 0.86 & 0.95 & 0.84 & 0.90 & 0.10 & 0.51 & 0.58 & 0.64 & 0.50 & 0.19 & 0.51 \\
\hline Model 6 & 1.05 & 0.97 & 0.97 & 0.83 & 0.96 & 1.00 & 0.90 & 0.96 & 0.90 & 0.94 & 0.09 & 0.43 & 0.29 & $\mid 0.44$ & 0.34 & 0.08 & 0.35 \\
\hline Model 7 & 1.06 & 1.09 & 1.14 & 1.07 & 1.09 & 1.00 & 0.90 & 0.93 & 0.89 & 0.93 & 0.11 & 0.47 & 0.41 & 0.48 & 0.40 & 0.03 & 0.37 \\
\hline Model 8 & 1.02 & 0.97 & 1.12 & 1.00 & 1.03 & 1.00 & 0.89 & 0.96 & 0.87 & 0.93 & 0.08 & 0.46 & 0.33 & $|0.51|$ & 0.38 & 0.06 & 0.37 \\
\hline Model 9 & 0.92 & 0.91 & 1.01 & 0.66 & 0.88 & 0.99 & 0.86 & 0.93 & 0.87 & 0.91 & 0.14 & 0.51 & 0.37 & 0.54 & 0.42 & 0.13 & 0.46 \\
\hline Obs1 & 1.00 & 0.99 & 0.97 & 1.07 & 1.01 & 1.00 & 0.99 & 0.98 & 0.98 & 0.99 & 0.03 & 0.13 & 0.18 & 0.21 & 0.16 & 0.04 & 0.23 \\
\hline Obs2 & 0.99 & 1.01 & 0.99 & 1.00 & 1.00 & 1.00 & 0.99 & 0.99 & 0.98 & 0.99 & 0.04 & 0.11 & 0.16 & 0.18 & 0.13 & 0.01 & 0.22 \\
\hline Obs3 & 1.01 & 1.02 & 1.10 & 0.97 & 1.02 & 1.00 & 1.00 & 0.97 & 0.99 & 0.99 & 0.04 & 0.09 & 0.27 & 0.16 & 0.17 & 0.05 & 0.24 \\
\hline
\end{tabular}

2008) and Global Precipitation Climatology Centre (GPCC) precipitation (Schneider et al., 2014). All observational data are available at $0.5^{\circ} \times 0.5^{\circ}$ resolution. We take the average of three pairs of SAT and precipitation values as the reference data in this study, unless stated otherwise. The observational uncertainty can be roughly estimated by comparing each observational estimate to the reference data (Xu et al., 2016). All datasets were regridded to a common resolution of $2.5^{\circ} \times 2.5^{\circ}$ using a box-averaging (bilinear interpolation) method that re-grids data to a coarse (fine) resolution. All datasets were weighted by the area of the grid cell to make the statistics more representative for the global mean values. Both the model and observational data are normalized by the rms value of each observed field before computing their statistics (Eqs. 19, 20).

Table 1 shows the various statistics of nine CMIP5 models in terms of the climatological mean summer (June-JulyAugust) SAT, precipitation, and the temporal standard deviation of SAT and precipitation over the global land area $\left(60^{\circ} \mathrm{S}-60^{\circ} \mathrm{N}\right)$. The standard deviation reflects the amplitude of interannual variation. The models can generally well simulate the climatological mean SAT characterized by the close correspondence of the rms values, high uncentered correlations, and small RMSDs between the model and observation. In contrast, models show a relatively poor performance in simulating other variables, i.e., climatological mean precipitation, standard deviations of SAT and precipitation. These statistics vary from one model to the next. It is difficult to compare the overall performance of various models because there are too many variables and models to distinguish one from another (Table 1). It is very useful to summarize the statistics of multiple variables with fewer indices, which enables an objective evaluation of the overall model performance in simulating multiple variables. To achieve this goal, we grouped the four normalized scalar fields into a fourdimensional vector field. Afterwards, we computed the statistical quantities, i.e., RMSL, VSC, and RMSVD, with the four-dimensional vector fields derived from model and observational data. As interpreted in Sect. 2, the RMSL (RMSVD) measures the overall rms values (RMSDs) of all scalar fields (Eqs. 3, 16). The VSC represents the weighted average of uncentered correlation coefficients across all scalar fields (Eq. 13). Thus, each model's performance in simulating multiple variables can be summarized by a single point that is determined by 12 statistical quantities ( 4 variables $\times 3$ statistics) derived from various scalar fields (Table 1, Fig. 2).

As shown in Fig. 2, the VSC varies from 0.90 to 0.94, indicating which models can better reproduce the overall spatial pattern of various variables and which cannot. For example, model 1 shows the maximum VSC, indicating that model 1 can generally better reproduce the spatial pattern of the four variables relative to other models. This can be confirmed by Table 1 . The uncentered pattern correlation coefficients for the four scalar fields are generally higher in model 1 than in the other models. Figure 2 also clearly shows which model overestimates or underestimates the overall rms 


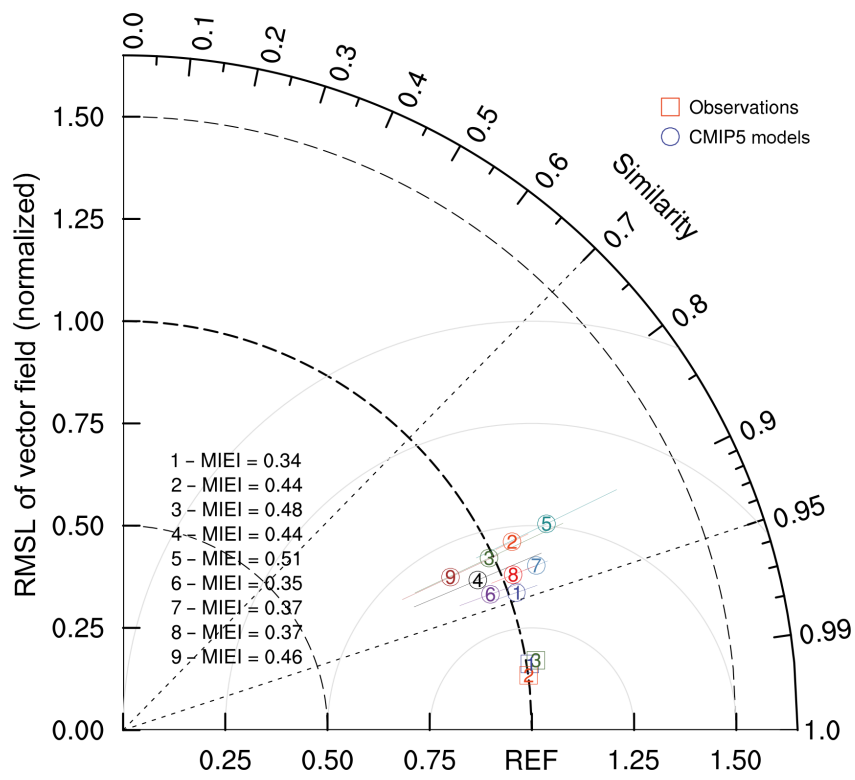

Figure 2. VFE diagram describing the normalized climatological mean SAT, precipitation, and interannual variabilities of SAT and precipitation over a land area between $60^{\circ} \mathrm{S}$ and $60^{\circ} \mathrm{N}$ simulated by nine CMIP5 models compared with three groups of SAT and precipitation data observed during the period from 1961 to 2000. The RMSL and the RMSVD have been normalized by dividing the RMSL derived from the observed data. The line segment centered at each plotted point along the azimuthal direction represents 2 standard deviations of the rms values of various fields. The value of the MIEI for each model is also shown in the diagram.

values. For example, models 5 and 7 overestimate the RMSLs of the four-dimensional vector fields, suggesting that both models generally overestimate the rms values of the four scalar fields. This can also be confirmed by Table 1, as model 5 clearly overestimates the rms values of Ta (1.43) and $\mathrm{Pa}$ (1.19) and slightly underestimates the rms values of $\mathrm{Tm}$ (0.99) and Pm (0.94). Model 7 overestimates all rms values $(1.06,1.09,1.14$, and 1.07) of the four variables. Thus, the RMSL of a constructed vector field can reasonably represent the overall performance of a model in reproducing rms values of multiple scalar fields. In contrast, model 9 clearly underestimates the RMSL of the vector field (Fig. 2). Correspondingly, three out of the four rms values of scalar fields are smaller than 1 for model 9 (Table 1). Similarly, the RMSVD between two vector fields can also reasonably represent the overall RMSDs of multiple scalar fields as shown in Fig. 2 and Table 1. Thus, one can evaluate the model performance in simulating multiple variables with three statistical quantities. The three statistical quantities represent different aspects of model performance, the knowledge of which can provide a more comprehensive model evaluation. The VFE diagram can clearly illustrate to what extent the overall RMSDs of various scalar fields (represented by the RMSVD) are attributable to the systematic difference in rms values (repre- sented by the RMSL) and how much is due to the poor pattern similarities (represented by $R_{\mathrm{v}}$ ).

Note that model performance does not change monotonically with the increase or decrease in rms values. Specifically, model performance improves as the normalized rms values approach 1 but decreases as the normalized rms values approach either zero or infinity. As defined in Eq. (3), the RMSL being equal to the sum of rms values of all components of a vector field. Thus, even if the modeled RMSL is equal to the observed one, it does not necessarily suggest that the model well reproduces the rms values of various scalar fields. This conclusion may result from the cancellation between the overestimated and the underestimated rms values. For example, as shown in Table 1, model 3 overestimates the rms values of Tm (1.05) and Ta (1.26) but underestimates the rms values of $\mathrm{Pm}(0.80)$ and $\mathrm{Pa}(0.77)$. However, the RMSL (0.99) is almost consistent with the observational estimate. Under such a circumstance, the RMSL misrepresents the model performance in simulating rms values of various scalar fields. To mitigate this shortcoming, one can add a line segment centered at each plotted point along the azimuthal direction (Fig. 2). The length of the line segment is equal to twice the standard deviation of rms values of multiple scalar fields. Thus, the length of the line segment can measure the dispersion of various rms values relative to their mean. A shorter line indicates that the rms values are close to the mean. In contrast, a longer line segment indicates that the rms values are spread out over a wider range. To measure the accuracy of modeled rms values to that of those observed, one can use the root mean square deviation of the rms values of various variables:

$\operatorname{RMSD}_{L}^{2}=\frac{1}{M} \sum_{i=1}^{M}\left(L_{a i}^{\star}-L_{b i}^{\star}\right)^{2}$,

where $L_{a i}^{\star}=\frac{1}{L_{b i}} \sqrt{\frac{1}{N} \sum_{j=1}^{N} a_{i j}^{2}}$ and $L_{b i}^{\star}=\frac{1}{L_{b i}} \sqrt{\frac{1}{N} \sum_{j=1}^{N} b_{i j}^{2}}$ are the rms values of the $i$ th normalized component of vector fields $\boldsymbol{A}$ and $\boldsymbol{B}$, respectively. With the support of Eq. (6), we have $L_{b i}^{\star}=1$ for all $i(1 \leq i \leq M)$. The $\operatorname{RMSD}_{L}^{2}$ can be further written as

$$
\begin{aligned}
\operatorname{RMSD}_{L}^{2} & =\frac{1}{M} \sum_{i=1}^{M}\left(L_{a i}^{\star}-1\right)^{2} \\
& =\frac{1}{M} \sum_{i=1}^{M} L_{a i}^{\star}{ }^{2}-\frac{2}{M} \sum_{i=1}^{M} L_{a i}^{\star}+1 \\
& =\frac{1}{M} \sum_{i=1}^{M}\left(\overline{L_{a}^{\star}}+L_{a i}^{\star}\right)^{2}-2 \overline{L_{a}^{\star}}+1 \\
& ={\overline{L_{a}^{\star}}}^{2}+\frac{1}{M} \sum_{i=1}^{M}{L_{a i}^{\star}}^{\prime 2}-2 \overline{L_{a}^{\star}}+1 \\
& =\left(\overline{L_{a}^{\star}}-1\right)^{2}+\sigma_{\mathrm{rms}}^{2},
\end{aligned}
$$


where $\overline{L_{a}^{\star}}$, and $L_{a i}^{\star \prime}$ are the mean and anomaly of $L_{a i}^{\star}$, respectively. The rms value of $L_{a i}^{\star}{ }^{\prime}$ is written as follows:

$\sigma_{\mathrm{rms}}=\left(\frac{1}{M} \sum_{i=1}^{M} L_{a i}^{\star \prime 2}\right)^{\frac{1}{2}}$.

$\sigma_{\mathrm{rms}}$ is the centered rms value or the standard deviation of $L_{a i}^{\star}$. Thus, the $\operatorname{RMSD}_{L}$ can be decomposed into the mean error and the variance of rms values of normalized scalar fields (Eq. 22). RMSD $_{L}$ measures the overall deviation of modeled rms values from the observed ones. The modeled rms values of various scalar fields are exactly equal to the corresponding observed ones only when the RMSD $L$ is equal to 0 .

\subsection{Multivariable integrated evaluation index for model performance}

In general, the model results get closer to the observational estimate as the RMSVD decreases. It is noteworthy that for a given VSC at a relatively low value, the RMSVD does not strictly decrease monotonically as the simulated RMSL approaches the observed one (Fig. 3). For example, model B shows the same VSC as that of model A but a smaller bias in the RMSL, which suggest that model B performs better than model A. However, the RMSVD is greater in model B than in model A (Fig. 3). Thus, the decrease in the RMSVD may not necessarily indicate an improvement in model performance. On the other hand, given the drawback of the RMSL in measuring the accuracy of rms values, the model skill score, defined based on the RMSL and VSC in Xu et al. (2016), is also not well suited for measuring the model performance in simulating multiple scalar fields. To better measure model performance, we define a multivariable integrated evaluation index (MIEI) based on the VFE diagram (Fig. 3):

$\mathrm{MIEI}^{2}=\mathrm{BC}^{2}+\mathrm{BG}^{2}$.

Based on the law of cosines, we have

$\mathrm{BG}^{2}=2-2 R_{\mathrm{v}}$.

Thus, the MIEI can be written as

$$
\begin{aligned}
\operatorname{MIEI}^{2} & =\operatorname{RMSD}_{L}^{2}+2\left(1-R_{\mathrm{V}}\right) \\
& =\sigma_{\text {rms }}^{2}+\left(\overline{L_{a}^{\star}}-1\right)^{2}+2\left(1-R_{\mathrm{v}}\right) .
\end{aligned}
$$

Clearly, the MIEI takes both the amplitudes and pattern similarities of various variables into account and therefore can provide a comprehensive evaluation of model performance (Eq. 24). In contrast to the RMSVD, the MIEI satisfies the monotonic property of an index with respect to model performance. Specifically, for any given $\sigma_{\mathrm{rms}}$ and $\overline{L_{a}^{\star}}$, the MIEI decreases monotonically with the increase in $R_{\mathrm{v}}$. For any given $\sigma_{\mathrm{rms}}$ and $R_{\mathrm{V}}$, the MIEI decreases monotonically as $\overline{L_{a}^{\star}}$ approaches 1 . For any given $\overline{L_{a}^{\star}}$ and $R_{\mathrm{v}}$, the MIEI decreases

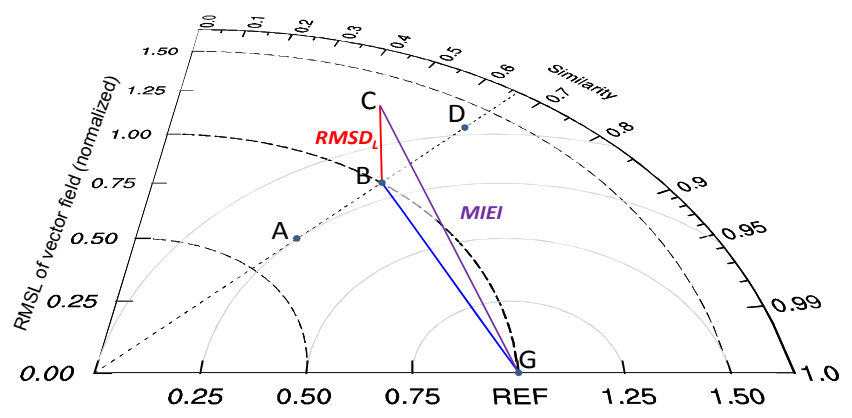

Figure 3. Schematic diagram displaying the relationship between the RMSVD, $\mathrm{RMSD}_{L}$, and MIEI. The points A, B, and D represent different models. The RMSD $\mathrm{R}_{L}$ measures the overall difference between the modeled rms values and the observed ones. The line segment $\mathrm{BC}$ is vertical with respect to the VFE diagram. The length of line segment $\mathrm{BG}$ is determined based on the vector field similarity, which measures the overall pattern similarity of various scalar fields relative to the observed ones. Thus, the MIEI index takes both the pattern similarities and the rms values of various scalar fields into account.

monotonically with the decrease in $\sigma_{\mathrm{rms}}$. The MIEI is equal to 0 only when $\sigma_{\mathrm{rms}}=0, \overline{L_{a}^{\star}}=1$, and $R_{\mathrm{V}}=1$, which define a perfect model. In other words, modeled multiple fields are exactly the same as the observed ones when the MIEI is equal to 0 .

As interpreted in Sect. 2, the RMSVD is determined based on the sum of quadratic RMSDs of various scalar fields (Eq. 16). Thus, the RMSVD is equivalent to the model climate performance index used in previous studies (e.g., Gleckler et al., 2008; Radić and Clarke, 2011; Chen and Sun, 2015). In general, both the RMSVD and MIEI can be used to measure the model performance. However, the MIEI is expected to provide a more accurate evaluation of model performance than the RMSVD. For example, model 3 shows a smaller RMSVD but a larger MIEI compared to model 2 (Table 1, Fig. 2). The RMSVD and MIEI give an opposite rank in the performance of models 2 and 3. Note that model 3 shows a much greater standard deviation of rms values $(0.20)$ than that of model $2(0.04)$, suggesting that model 3 poorly simulates the relative amplitude of the four variables. Such information is not considered by the RMSVD but can be captured by the MIEI (Eqs. 18, 24). The values of the MIEI derived from various models are also shown in Fig. 2. A smaller MIEI generally indicates a better performance of the climate model. For example, models 1 and 6 show smaller MIEIs than the other models. Models 1 and 6 show higher VSC values and a close correspondence of rms values with the observed ones (Table 1, Fig. 2). The MIEI can serve as an index to determine the rank of climate model performance in simulating multiple fields. In comparison with the MIEI, the VFE diagram can provide a more detailed evaluation of the model performance by explicitly showing mul- 
tiple statistics, i.e., pattern similarity, rms values and their variances, and RMSVD.

The issue of how to take the observational uncertainties into account is of particular importance in model evaluation and ranking, especially when more and more observational datasets provide estimates of the observational uncertainty. The statistics derived from each group of observational estimates are also shown in Table 1, which can roughly quantify the observational uncertainties and its impact on model evaluation. Generally, the colors are clearly lighter for the statistics of individual observed variables in contrast to the modeled variables (Table 1). This indicates that the observational uncertainties are relatively small and should have less impact on the evaluation of model performance. To further quantify the impacts of observational uncertainty on ranking model performance, we calculate the MIEIs of various climate models by taking each group of observational estimates as the reference data. Three groups of observational estimates generate three groups of MIEIs. Afterwards, we calculate Spearman's rank correlation coefficient of each group of MIEIs with those derived from models and the ensemble mean of multiple observational estimates. The Spearman's rank correlation coefficients are $0.996,0.996$, and 0.904 , respectively, suggesting that the ranks are very close to each other no matter which group of observational estimates is used as reference data. Thus, the observational uncertainty should have less impact on ranking model performance in this case. One can use the average of Spearman's rank correlation coefficients to quantify the consistency of various ranks when a number of observational estimates are available.

\section{Summary and discussion}

The MVIE method proposed here provides a concise way of representing the multiple statistics of multiple fields on a two-dimensional plot, i.e., the VFE diagram. The VFE diagram includes three statistical quantities, i.e., RMSL, VSC, and RMSVD, representing different aspects of model performance. Specifically, the RMSL (RMSVD) represents the total mean value and variance (total RMSDs) of all scalar fields. The VSC measures the overall pattern similarity across all scalar fields. As shown in the example, each of the three statistical quantities can reasonably represent the corresponding statistics of multiple scalar fields. Moreover, the VFE diagram can illustrate how much the overall RMSD of various fields is attributable to the difference in rms values and how much is due to poor pattern similarity. Thus, one can summarize multiple statistics of multiple variables for various models in a diagram and facilitate the intercomparison of model performance in simulating multiple variables. The MVIE method can be applied to spatial and/or temporal fields. It can also simultaneously evaluate various temporal variabilities simulated by models, e.g., climatological mean state and the amplitude of interannual variability as shown in Sect. 3.2. Based on the VFE diagram, we also developed a MIEI which takes the amplitude and pattern similarity of multiple fields into account. The MIEI satisfies the criterion that a model performance index should vary monotonically as the model performance improves. The MIEI provides a more concise evaluation than the VFE diagram of model performance in simulating multiple fields.

The statistical metrics presented in this paper can be divided into three different levels and their relationships are summarized in a pyramid chart (Fig. 4). The first level of metrics, i.e., correlation coefficient, rms value, and RMSD, measures model performance in terms of individual variables. These metrics can be illustrated by a table of metrics (Table 1), which can provide detailed information on model performance in simulating individual variables but cannot give a quantitative evaluation of the overall model performance in simulating multiple fields. The second level of metrics, i.e., the VSC, RMSL, standard deviation of rms values, and RMSVD, is derived from the first level of metrics and represents the overall statistics of multiple variables. The second level of metrics can be presented as a VFE diagram, which provides an integrated evaluation of model performance in terms of simulating multiple fields. The MIEI belongs to the third level of metrics, which is defined based on the VFE diagram. The MIEI further summarizes the three statistical quantities of the VFE diagram into a single index and can be used to rank the performance of various climate models. A higher level of metrics provides a more concise evaluation of model performance compared to a lower level of metrics, which facilitates model intercomparison. Unavoidably, the higher level of metrics loses detailed statistical information in contrast to the lower level of metrics. To provide a more comprehensive evaluation of model performance, one can show the VFE diagram together with a table of statistical metrics (Table 1) or other model performance metrics as needed.

As shown in Sect. 2, the VFE diagram can be constructed by using uncentered statistics, which are computed using the full scalar fields, including both mean and anomaly. The VFE diagram can also be constructed by using centered statistics (Appendix A). The centered RMSL of a vector represents the overall variance of all components of a vector field (Eq. A3). The centered VSC can be interpreted as weighted average of Pearson's correlation coefficients, which measures the overall pattern similarity across all paired anomaly fields (Eq. A9). The centered RMSVD measures the sum of centered RMSDs across all paired components between two vector fields (Eq. A12). The type of statistics, i.e., centered or uncentered statistics, that should be used depends on the application. The uncentered statistics should be used if both the mean and anomaly need to be evaluated. In contrast, the centered statistics should be used if the anomaly fields are the primary concern. The centered correlations alone are not sufficient for detection studies (Legates and Davis, 1997). It has 


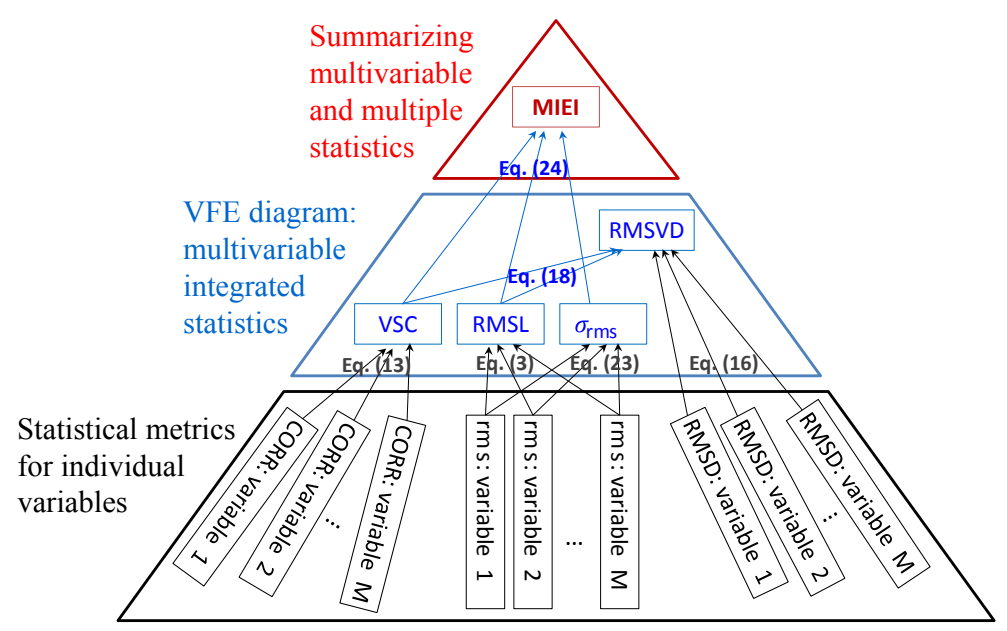

Figure 4. Pyramid chart showing the relationship between three levels of metrics. The first level of metrics, i.e., correlation coefficient (CORR), rms value, and RMSD, measures the model performance in terms of individual variables. The second level of metrics, i.e., VSC, RMSL, standard deviation of rms values $\left(\sigma_{\mathrm{rms}}\right)$, and RMSVD, is derived from the first level of metrics and summarizes the overall performance of a climate model in simulating multiple fields. The MIEI further summarizes the VSC, RMSL, and $\sigma_{\text {rms }}^{2}$ into a single index to rank various climate models in terms of simulating multiple fields.

been argued that the uncentered statistics are better suited for detection because they incorporate the response of the mean value. In contrast, the centered statistics are more appropriate for attribution because they better measure the similarity between spatial patterns (Hegerl et al., 2001). The VFE diagram provides us flexibility in model evaluation. In terms of model evaluation aimed at a detection study, one can compute the uncentered statistics with full fields. In contrast, one can use centered statistics by computing the statistical quantities with vector anomaly fields if an attribution study is the major concern of the model evaluation.

In practice, one may want to weight different fields based on their relative importance. If some variables to be evaluated are dependent on each other, e.g., skin temperature and surface air temperature, one may also want to weight these variables properly because the dependent variables contain redundant information. Otherwise, the evaluation of equally weighted variables may overestimate the importance of the dependent variables. Determining the weight coefficient depends on the application and therefore is beyond the scope of this study. Here, we only discuss how the weight can be considered in the multivariable integrated evaluation (Appendix B). The MVIE method presented in this study requires the normalization of each modeled and observed variable by dividing the corresponding rms value of the observed variable (Eqs. 19, 20). Therefore, one should weight different variables after the normalization (Eqs. B1, B2); otherwise the normalization process will remove the weight coefficient. Weighting each normalized field leads to a quadratic weighting of the quadratic rms values, quadratic RMSDs, and correlation coefficients (Eqs. B1, B5, B8, B11).
The VFE diagram and MIEI may also provide some guidance in weighting various climate models to constrain future climate projection. A recent study suggested that model weighting should take both model performance and model interdependencies into account to improve climate projections (Knutti et al., 2017). The VFE diagram can summarize model performance in terms of multiple statistics of multiple fields, on one hand. On the other hand, the VFE diagram can also clearly show the differences between model and observation as well as the differences between various models. This information provided by the VFE diagram may be used in weighting climate models, which warrant further studies.

Code availability. The code used in the production of Fig. 2 and Table 1 is available in the Supplement.

Data availability. CRU data are provided by Climatic Research Unit from their web site at https://crudata.uea.ac.uk/cru/data/hrg/ (last access: 16 October 2017). UDel_AirT_Precip, GPCC precipitation data (Schneider et al., 2011), and GHCN gridded v3 data are provided by the NOAA/OAR/ESRL PSD (Boulder, Colorado, USA) from their web site at https://www.esrl.noaa.gov/psd/data/ gridded/ (last access: 16 October 2017). 


\section{Appendix A: Decomposition of RMSL, VSC, and RMSVD}

To further interpret the RMSL, VSC, and RMSVD, we break down the full vector fields $\boldsymbol{A}$ and $\boldsymbol{B}$ into the mean and anomaly:

$$
\begin{gathered}
A_{j}=\bar{A}+A_{j}^{\prime}=\left(\overline{a_{1}}+a_{1 j}^{\prime}, \overline{a_{2}}+a_{2 j}^{\prime}, \cdots, \overline{a_{M}}+a_{M j}^{\prime}\right) ; \\
\quad j=1,2, \ldots, N \\
B_{j}=\bar{B}+B_{j}^{\prime}=\left(\overline{b_{1}}+b_{1 j}^{\prime}, \overline{b_{2}}+b_{2 j}^{\prime}, \cdots, \overline{b_{M}}+b_{M j}^{\prime}\right) ; \\
\quad j=1,2, \ldots, N
\end{gathered}
$$

where

$\bar{A}=\left(\overline{a_{1}}, \overline{a_{2}}, \cdots, \overline{a_{M}}\right)$,

$\bar{B}=\left(\overline{b_{1}}, \overline{b_{2}}, \cdots, \overline{b_{M}}\right)$,

$A_{j}^{\prime}=\left(a_{1 j}^{\prime}, a_{2 j}^{\prime}, \cdots, a_{M j}^{\prime}\right)$,

$B_{j}^{\prime}=\left(b_{1 j}^{\prime}, b_{2 j}^{\prime}, \cdots, b_{M j}^{\prime}\right)$,

$\overline{a_{i}}=\frac{1}{N} \sum_{j=1}^{N} a_{i j} ; \quad i=1,2, \ldots, M$

$\overline{b_{i}}=\frac{1}{N} \sum_{j=1}^{N} b_{i j} ; \quad i=1,2, \ldots, M$

$a_{i j}^{\prime}=a_{i j}-\overline{a_{i}} ; \quad i=1,2, \ldots, M$

$b_{i j}^{\prime}=b_{i j}-\overline{b_{i}} ; \quad i=1,2, \ldots, M$.

The squared RMSL of vector field $\boldsymbol{A}$ is written as follows:

$$
\begin{aligned}
L_{A}^{2} & =\frac{1}{N} \sum_{j=1}^{N}\left\|A_{j}\right\|^{2} \\
& =\frac{1}{N} \sum_{j=1}^{N} \sum_{i=1}^{M}\left(\overline{a_{i}}+a_{i j}^{\prime}\right)^{2} \\
& =\frac{1}{N} \sum_{j=1}^{N} \sum_{i=1}^{M}{\overline{a_{i}}}^{2}+\frac{1}{N} \sum_{j=1}^{N} \sum_{i=1}^{M} a_{i j}^{\prime}+\frac{1}{N} \sum_{j=1}^{N} \sum_{i=1}^{M} 2 \overline{a_{i}} a_{i j}^{\prime} .
\end{aligned}
$$

Given $\sum_{j=1}^{N} a_{i j}^{\prime}=0, L_{A}^{2}$ can be written as

$$
\begin{aligned}
L_{A}^{2} & =\frac{1}{N} \sum_{j=1}^{N} \sum_{i=1}^{M} \bar{a}_{i}^{2}+\frac{1}{N} \sum_{j=1}^{N} \sum_{i=1}^{M} a_{i j}^{\prime 2} \\
& =\frac{1}{N} \sum_{j=1}^{N}\|\overline{\boldsymbol{A}}\|^{2}+\frac{1}{N} \sum_{j=1}^{N}\left\|\boldsymbol{A}_{j}^{\prime}\right\|^{2} \\
& =L_{\bar{A}}^{2}+L_{A^{\prime}}^{2},
\end{aligned}
$$

where

$$
L_{\bar{A}}=\left(\frac{1}{N} \sum_{j=1}^{N}\|\overline{\boldsymbol{A}}\|^{2}\right)^{\frac{1}{2}}=\|\overline{\boldsymbol{A}}\|=\left(\sum_{i=1}^{M}{\overline{a_{i}}}^{2}\right)^{\frac{1}{2}}
$$

is the RMSL of the mean vector field,

$$
L_{A^{\prime}}=\left(\frac{1}{N} \sum_{j=1}^{N}\left\|\boldsymbol{A}_{j}^{\prime}\right\|^{2}\right)^{\frac{1}{2}}=\left(\sum_{i=1}^{M} \sigma_{a i}^{2}\right)^{\frac{1}{2}}
$$

is the RMSL of the vector anomaly field, and

$$
\sigma_{a i}=\left(\frac{1}{N} \sum_{j=1}^{N}{a_{i j}^{\prime}}^{2}\right)^{\frac{1}{2}} ; \quad i=1,2, \ldots, M
$$

is the centered rms value (or standard deviation) of the $i$ th component of vector field $\boldsymbol{A}$.

Equation (A1) can be written as

$$
L_{A}^{2}=L_{\bar{A}}^{2}+L_{A^{\prime}}^{2}=\sum_{i=1}^{M}\left({\overline{a_{i}}}^{2}+\sigma_{a i}^{2}\right) .
$$

The RMSL of vector field $\boldsymbol{A}, L_{A}$, measures the overall mean value and variance of all components of the vector field.

Similarly, we have

$L_{B}^{2}=L_{\bar{B}}^{2}+L_{B^{\prime}}^{2}=\sum_{i=1}^{M}\left({\overline{b_{i}}}^{2}+\sigma_{b i}^{2}\right)$,

where

$$
\sigma_{b i}=\left(\frac{1}{N} \sum_{j=1}^{N} b_{i j}^{\prime 2}\right)^{\frac{1}{2}} ; \quad i=1,2, \ldots, M
$$

is the centered rms value (or standard deviation) of the $i$ th component of vector field $\boldsymbol{B}$.

With the support of Eq. (13), the VSC can be written as

$$
\begin{aligned}
R_{\mathrm{V}}= & \frac{1}{N L_{A} L_{B}} \sum_{j=1}^{N} \sum_{i=1}^{M}\left(\overline{a_{i}}+a_{i j}^{\prime}\right)\left(\overline{b_{i}}+b_{i j}^{\prime}\right) \\
= & \frac{1}{N L_{A} L_{B}} \sum_{j=1}^{N} \sum_{i=1}^{M}\left(\overline{a_{i}} \overline{b_{i}}+\overline{a_{i}} b_{i j}^{\prime}+\overline{b_{i}} a_{i j}^{\prime}+a_{i j}^{\prime} b_{i j}^{\prime}\right) \\
= & \frac{1}{N L_{A} L_{B}} \sum_{i=1}^{M} \\
& \left(\sum_{j=1}^{N} \overline{a_{i}} \overline{b_{i}}+\sum_{j=1}^{N} \overline{a_{i}} b_{i j}^{\prime}+\sum_{j=1}^{N} \overline{b_{i}} a_{i j}^{\prime}+\sum_{j=1}^{N} a_{i j}^{\prime} b_{i j}^{\prime}\right) .
\end{aligned}
$$

Given that $\sum_{j=1}^{N} a_{i j}^{\prime}=\sum_{j=1}^{N} b_{i j}^{\prime}=0$ for all $i(1 \leq i \leq M)$, we obtain

$$
\begin{aligned}
R_{\mathrm{v}} & =\frac{1}{N L_{A} L_{B}} \sum_{i=1}^{M}\left(\sum_{j=1}^{N} \overline{a_{i}} \overline{b_{i}}+\sum_{j=1}^{N} a_{i j}^{\prime} b_{i j}^{\prime}\right) \\
& =\frac{L_{\bar{A}} L_{\bar{B}}}{L_{A} L_{B}} R_{\overline{\mathrm{v}}}+\frac{L_{A^{\prime}} L_{B^{\prime}}}{L_{A} L_{B}} R_{\mathrm{v}^{\prime}},
\end{aligned}
$$


where

$$
\begin{aligned}
& R_{\overline{\mathrm{v}}}=\frac{1}{N L_{\bar{A}} L_{\bar{B}}} \sum_{j=1}^{N} \sum_{i=1}^{M} \overline{a_{i}} \overline{b_{i}}=\frac{1}{L_{\bar{A}} L_{\bar{B}}} \sum_{i=1}^{M} \overline{a_{i}} \overline{b_{i}} \\
& R_{\mathrm{v}^{\prime}}=\frac{1}{N L_{A^{\prime}} L_{B^{\prime}}} \sum_{j=1}^{N} \sum_{i=1}^{M} a_{i j}^{\prime} b_{i j}^{\prime} .
\end{aligned}
$$

Given the Cauchy-Schwarz inequality, Eq. (A7) can be rewritten as

$R_{\overline{\mathrm{v}}}^{2}=\frac{\left(\sum_{i=1}^{M} \overline{a_{i}} \overline{b_{i}}\right)^{2}}{\left(L_{\bar{A}} L_{\bar{B}}\right)^{2}} \leq \frac{\sum_{i=1}^{M}{\overline{a_{i}}}^{2} \sum_{i=1}^{M}{\overline{b_{i}}}^{2}}{\left(L_{\bar{A}} L_{\bar{B}}\right)^{2}}=\frac{L_{\bar{A}}^{2} L_{\bar{B}}^{2}}{\left(L_{\bar{A}} L_{\bar{B}}\right)^{2}}=1$.

$R_{\overline{\mathrm{v}}}$ reaches its maximum value of 1 when $\frac{\overline{a_{1}}}{\overline{b_{1}}}=\frac{\overline{a_{2}}}{\overline{b_{2}}}=\ldots=$ $\frac{\overline{a_{M}}}{\overline{b_{M}}}>0$. In contrast, $R_{\overline{\mathrm{V}}}$ reaches its minimum value of -1 when $\frac{\overline{a_{1}}}{\overline{b_{1}}}=\frac{\overline{a_{2}}}{\overline{b_{2}}}=\ldots=\frac{\overline{a_{M}}}{\overline{b_{M}}}<0 . R_{\overline{\mathrm{v}}}$ measures the extent of correlation between modeled and observed mean values across all components of two vector fields.

Equation (A8) can be rewritten as

$$
\begin{aligned}
R_{\mathrm{V}^{\prime}} & =\frac{1}{N L_{A^{\prime}} L_{B^{\prime}}} \sum_{j=1}^{N} \sum_{i=1}^{M} \frac{a_{i j}^{\prime} b_{i j}^{\prime}}{\sigma_{a i} \sigma_{b i}} \sigma_{a i} \sigma_{b i} \\
& =\frac{1}{N L_{A^{\prime}} L_{B^{\prime}}} \sum_{i=1}^{M} \sum_{j=1}^{N} \frac{a_{i j}^{\prime} b_{i j}^{\prime}}{\sigma_{a i} \sigma_{b i}} \sigma_{a i} \sigma_{b i} \\
& =\frac{1}{L_{A^{\prime}} L_{B^{\prime}}} \sum_{i=1}^{M}\left(\sigma_{a i} \sigma_{b i} \frac{1}{N} \sum_{j=1}^{N} \frac{a_{i j}^{\prime} b_{i j}^{\prime}}{\sigma_{a i} \sigma_{b i}}\right) \\
& =\frac{1}{L_{A^{\prime}} L_{B^{\prime}}} \sum_{i=1}^{M} \sigma_{a i} \sigma_{b i} r_{i},
\end{aligned}
$$

where $\sigma_{a i}$ and $\sigma_{b i}$ are the centered rms values (or standard deviation) of the $i$ th component of vector field $\boldsymbol{A}$ and $\boldsymbol{B}$, respectively.

$r_{i}=\frac{1}{N} \sum_{j=1}^{N} \frac{a_{i j}^{\prime} b_{i j}^{\prime}}{\sigma_{a i} \sigma_{b i}}$

represents the centered correlation coefficients between the $i$ th paired components of vector fields $\boldsymbol{A}$ and $\boldsymbol{B} . R_{\mathrm{v}^{\prime}}$ can be interpreted as a weighted average of the centered correlation coefficients across all paired components between two vector fields. The weight coefficients are proportional to the product of standard deviations between paired variables. Clearly, the VSC is simultaneously determined based on the correlation of various mean fields and the overall correlation of anomaly fields across all paired components between two vector fields (Eqs. A6, A7, A9).
The RMSVD between two vector fields can also be represented by the mean and anomaly fields:

$$
\begin{aligned}
\operatorname{RMSVD}^{2}= & \frac{1}{N} \sum_{j=1}^{N}\left\|\boldsymbol{A}_{j}-\boldsymbol{B}_{j}\right\|^{2} \\
= & \frac{1}{N} \sum_{j=1}^{N} \sum_{i=1}^{M}\left(\overline{a_{i}}+a_{i j}^{\prime}-\overline{b_{i}}-b_{i j}^{\prime}\right)^{2} \\
= & \frac{1}{N} \sum_{i=1}^{M} \sum_{j=1}^{N}\left(\overline{a_{i}}-\overline{b_{i}}+a_{i j}^{\prime}-b_{i j}^{\prime}\right)^{2} \\
= & \frac{1}{N} \sum_{i=1}^{M}\left(\sum_{j=1}^{N}\left(\overline{a_{i}}-\overline{b_{i}}\right)^{2}+\sum_{j=1}^{N}\left(a_{i j}^{\prime}-b_{i j}^{\prime}\right)^{2}\right. \\
& \left.+2\left(\overline{a_{i}}-\overline{b_{i}}\right) \sum_{j=1}^{N}\left(a_{i j}^{\prime}-b_{i j}^{\prime}\right)\right) .
\end{aligned}
$$

Given that $\sum_{j=1}^{N} a_{i j}^{\prime}=\sum_{j=1}^{N} b_{i j}^{\prime}=0$ for all $i(1 \leq i \leq M)$, we obtain

$$
\begin{aligned}
\operatorname{RMSVD}^{2} & =\frac{1}{N} \sum_{i=1}^{M}\left(\sum_{j=1}^{N}\left(\overline{a_{i}}-\overline{b_{i}}\right)^{2}+\sum_{j=1}^{N}\left(a_{i j}^{\prime}-b_{i j}^{\prime}\right)^{2}\right) \\
& =\frac{1}{N} \sum_{j=1}^{N} \sum_{i=1}^{M}\left(\overline{a_{i}}-\overline{b_{i}}\right)^{2}+\frac{1}{N} \sum_{j=1}^{N} \sum_{i=1}^{M}\left(a_{i j}^{\prime}-b_{i j}^{\prime}\right)^{2} \\
& =\frac{1}{N} \sum_{j=1}^{N}|\overline{\boldsymbol{A}}-\overline{\boldsymbol{B}}|^{2}+\frac{1}{N} \sum_{j=1}^{N}\left|\boldsymbol{A}_{j}^{\prime}-\boldsymbol{B}_{j}^{\prime}\right|^{2} \\
& =(\mathrm{RMSVDm})^{2}+(\mathrm{RMSVDa})^{2},
\end{aligned}
$$

where

$(\mathrm{RMSVDm})^{2}=\sum_{i=1}^{M}\left(\overline{a_{i}}-\overline{b_{i}}\right)^{2}$

is the RMSVD between mean vector fields $\overline{\boldsymbol{A}}$ and $\overline{\boldsymbol{B}}$, which represents the mean difference of all fields.

$$
\begin{aligned}
\left(\operatorname{RMSVDa}^{2}\right. & =\sum_{i=1}^{M}\left(\frac{1}{N} \sum_{j=1}^{N}\left(a_{i j}^{\prime}-b_{i j}^{\prime}\right)^{2}\right) \\
& =\sum_{i=1}^{M} \operatorname{RMSD}_{i}^{\prime 2}
\end{aligned}
$$

is the centered RMSVD between two vector fields, which represents the overall RMSD across all paired components of vector anomaly fields $\boldsymbol{A}^{\prime}$ and $\boldsymbol{B}^{\prime}$. From the viewpoint of MVIE, the RMSVD can be interpreted as the overall mean difference of all fields plus the overall RMSD of all anomaly fields.

The statistics can be computed based on the full vector fields or anomaly vector fields depending on the concern of 
evaluation. The statistical quantities, i.e., RMSL, VSC, and RMSVD, computed based on the full vector fields represent the uncentered pattern statistics, which include the statistics from both the mean and anomaly fields. Alternatively, three statistics can also be computed based on the anomaly fields, yielding centered statistics, which only measure the anomaly fields. The full vector fields should be used if both the mean and anomaly need to be evaluated. In contrast, the anomaly vector fields should be used if anomaly fields are the primary concern.

\section{Appendix B: Weighted multivariable integrated evaluation with VFE diagram}

In terms of model evaluation, one may care for some variables more than other variables, although all variables are of great concern. In such a circumstance, it would be useful to weight different variables to make the VSC, RMSL, and RMSVD more sensitive to some variables than to others. Without loss of generality, the weighted- and normalizedvector fields $\boldsymbol{A}^{\mathrm{w}}$ and $\boldsymbol{B}^{\mathrm{w}}$ can be written as a pair of vector sequences:

$$
\begin{aligned}
\boldsymbol{A}_{j}^{\mathrm{w}} & =\boldsymbol{w} \cdot \boldsymbol{A}_{j}^{\star}=\left(w_{1} a_{1 j}^{\star}, w_{2} a_{2 j}^{\star}, \ldots, w_{M} a_{M j}^{\star}\right) ; \\
j & =1,2, \ldots, N \\
\boldsymbol{B}_{j}^{\mathrm{w}} & =\boldsymbol{w} \cdot \boldsymbol{B}_{j}^{\star}=\left(w_{1} b_{1 j}^{\star}, w_{2} b_{2 j}^{\star}, \ldots, w_{M} b_{M j}^{\star}\right) ; \\
j & =1,2, \ldots, N,
\end{aligned}
$$

where $a_{i j}^{\star}$ and $b_{i j}^{\star}(1 \leq i \leq M)$ are the same as in Eqs. (19) and (20). $w_{i}$ is the weight coefficient for the $i$ th component of the vector field and satisfies the constraint

$$
\sum_{i=1}^{M} w_{i}=M
$$

Note that the weighting should be applied to the normalized model and observational data (Eqs. B1, B2). Otherwise, the normalization would remove the weight coefficient (Eqs. 19 and 20). Based on Eq. (3), the square of the RMSL of the normalized vector field $\boldsymbol{A}^{\star}$ can be written as follows:

$L_{A}^{\star 2}=\sum_{i=1}^{M} L_{a i}^{\star 2}$,

where $L_{a i}^{\star}=\left(\frac{1}{N} \sum_{j=1}^{N} a_{i j}^{\star}\right)^{\frac{1}{2}}$ denotes the rms value of the $i$ th component of the normalized vector field $\boldsymbol{A}^{\star}$. Similarly, the quadratic RMSL of weighted- and normalized-vector fields can be written as follows:

$L_{A}^{\mathrm{w} 2}=\sum_{i=1}^{M} L_{a i}^{\mathrm{w} 2}$ where $L_{a i}^{\mathrm{w}}=\left(\frac{1}{N} \sum_{j=1}^{N} w_{i}^{2} a_{i j}^{\star 2}\right)^{\frac{1}{2}}=w_{i} L_{a i}^{\star}$ is the rms value of the $i$ th component of vector field $\boldsymbol{A}^{\mathrm{w}}$. With the support of Eqs. (B1), (B3), and (B4), it is easy to obtain

$L_{A}^{\mathrm{w} 2}=\sum_{i=1}^{M} L_{a i}^{\mathrm{w} 2}=\sum_{i=1}^{M} w_{i}^{2} L_{a i}^{\star 2}$.

The RMSL of vector field $\boldsymbol{A}^{\mathrm{w}}$ is determined based on the weighted rms values across all components of the vector field. The contribution of the $i$ th rms value, $L_{a i}^{\star}$, to the quadratic RMSL of the vector field is weighted by $w_{i}^{2}$. The rms value accounts for more of the RMSL when its weight coefficient is greater.

Based on Eq. (16), the square of the RMSVD between normalized vector fields $\boldsymbol{A}^{\star}$ and $\boldsymbol{B}^{\star}$ can be written as follows:

$\operatorname{RMSVD}^{\star 2}=\sum_{i=1}^{M} \operatorname{RMSD}_{i}^{\star 2}$,

where $\operatorname{RMSD}_{i}=\left(\frac{1}{N} \sum_{j=1}^{N}\left(a_{i j}^{\star}-b_{i j}^{\star}\right)^{2}\right)^{\frac{1}{2}}$ is the RMSD of the $i$ th paired components between normalized vector fields $\boldsymbol{A}^{\star}$ and $\boldsymbol{B}^{\star}$. Similarly, the square of the RMSVD between weighted vector fields $\boldsymbol{A}^{\mathrm{w}}$ and $\boldsymbol{B}^{\mathrm{w}}$ can be written as follows:

$\operatorname{RMSVD}^{\mathrm{w} 2}=\sum_{i=1}^{M}\left(\mathrm{RMSD}_{i}^{\mathrm{w}}\right)^{2}$.

$\operatorname{RMSD}_{i}^{\mathrm{w}}=\left(\frac{1}{N} \sum_{j=1}^{N}\left(w_{i} a_{i j}^{\star}-w_{i} b_{i j}^{\star}\right)^{2}\right)^{\frac{1}{2}}$ is the RMSD of the $i$ th paired components between weighted vector fields $\boldsymbol{A}^{\mathrm{w}}$ and $\boldsymbol{B}^{\mathrm{w}}$. With the aid of Eqs. (B1), (B2), (B6), and (B7), we obtain

$\left(\operatorname{RMSVD}^{\mathrm{w}}\right)^{2}=\sum_{i=1}^{M}\left(\operatorname{RMSD}_{i}^{\mathrm{W}}\right)^{2}=\sum_{i=1}^{M} w_{i}^{2} \mathrm{RMSD}_{i}^{\star 2}$.

The RMSVD between two vector fields is determined based on the weighted RMSDs across all paired components of two vector fields. The contribution of the $i$ th RMSD to the quadratic RMSVD between two vector fields is weighted by $w_{i}^{2}$.

Based on Eq. (13), the VSC between normalized vector fields $\boldsymbol{A}^{\star}$ and $\boldsymbol{B}^{\star}$ can be written as follows:

$R_{\mathrm{v}}^{\star}=\frac{1}{L_{A}^{\star} \cdot L_{B}^{\star}} \sum_{i=1}^{M} L_{a i}^{\star} L_{b i}^{\star} R_{u i}^{\star}$,

where $L_{a i}^{\star}=\left(\frac{1}{N} \sum_{j=1}^{N} a_{i j}^{\star 2}\right)^{\frac{1}{2}}$ and $L_{b i}^{\star}=\left(\frac{1}{N} \sum_{j=1}^{N} b_{i j}^{\star 2}\right)^{\frac{1}{2}}$ are the rms values for the $i$ th normalized components of vector 
fields $\boldsymbol{A}$ and $\boldsymbol{B}$, respectively. $R_{u i}^{\star}=\frac{\frac{1}{N} \sum_{j=1}^{N} a_{i j}^{\star} b_{i j}^{\star}}{L_{a i}^{\star} L_{b i}^{\star}}$ is the uncentered correlation coefficient for the $i$ th components between two vector fields. Similarly, the VSC between weighted fields can be rewritten as

$$
R_{\mathrm{v}}^{\mathrm{w}}=\frac{1}{L_{A}^{\mathrm{w}} \cdot L_{B}^{\mathrm{w}}} \sum_{i=1}^{M} L_{a i}^{\mathrm{w}} L_{b i}^{\mathrm{w}} R_{u i}^{\mathrm{w}}
$$

where $L_{a i}^{\mathrm{w}}, L_{b i}^{\mathrm{w}}$, and $R_{u i}^{\mathrm{w}}$ are the same as $L_{a i}^{\star}, L_{b i}^{\star}$, and $R_{u i}^{\star}$, respectively, except they are computed based on the weighted vector fields $\boldsymbol{A}^{\mathrm{w}}$ and $\boldsymbol{B}^{\mathrm{w}}$. With the aid of Eqs. (B1), (B2), (B9), and (B10), we obtain

$$
R_{\mathrm{v}}^{\mathrm{W}}=\frac{1}{L_{A}^{\mathrm{W}} \cdot L_{B}^{\mathrm{W}}} \sum_{i=1}^{M} w_{i}^{2} L_{a i}^{\star} L_{b i}^{\star} R_{u i}^{\star}
$$

The VSC is determined based on the sum of the products of the uncentered correlation coefficients and the rms values. The contribution of the $i$ th product term, $L_{a i}^{\star} L_{b i}^{\star} R_{u i}^{\star}$, to the VSC is weighted by $w_{i}^{2}$. 


\section{The Supplement related to this article is available online at https://doi.org/10.5194/gmd-10-3805-2017- supplement.}

Author contributions. ZX devised the evaluation method and wrote the paper. All of the authors discussed the results and commented on the paper.

Competing interests. The authors declare that they have no conflict of interest.

Acknowledgements. We acknowledge the World Climate Research Programme's Working Group on Coupled Modelling, which is responsible for CMIP, and we thank the climate modeling groups for producing and making available their model output. The study was supported jointly by the National Key Research and Development Program of China (2016YFA0600403), the Major Research Plan of the National Science Foundation of China (91637103), and the National Science Foundation of China Grant (41675080, 41675105). This work was also supported by the Jiangsu Collaborative Innovation Center for Climate Change.

Edited by: Klaus Gierens

Reviewed by: two anonymous referees

\section{References}

Chen, H. and Sun, J.: Assessing model performance of climate extremes in China: an intercomparison between CMIP5 and CMIP3, Climatic Change, 129, 197-211, 2015.

Eyring, V., Gleckler, P. J., Heinze, C., Stouffer, R. J., Taylor, K. E., Balaji, V., Guilyardi, E., Joussaume, S., Kindermann, S., Lawrence, B. N., Meehl, G. A., Righi, M., and Williams, D. N.: Towards improved and more routine Earth system model evaluation in CMIP, Earth Syst. Dynam., 7, 813-830, https://doi.org/10.5194/esd-7-813-2016, 2016.

Fan, Y. and van den Dool, H.: A global monthly land surface air temperature analysis for 1948-present, J. Geophys. Res., 113, D01103, https://doi.org/10.1029/2007JD008470, 2008.

Fu, C., Wang, S., Xiong, Z., Gutowski, W. J., Lee, D.-K., McGregor, J. L., Sato, Y., Kato, H., Kim, J.-W., and Suh, M.-S.: Regional Climate Model Intercomparison Project for Asia, B. Am. Meteorol. Soc., 86, 257-266, 2005.

Giorgi, F. and Gutowski, W. J.: Regional Dynamical Downscaling and the CORDEX Initiative, Annu. Rev. Environ. Res., 40, 467490, 2015.

Gleckler, P. J., Taylor, K. E., and Doutriaux, C.: Performance metrics for climate models, J. Geophys. Res., 113, D06104, https://doi.org/10.1029/2007JD008972, 2008.

Harris, I., Jones, P. D., Osborn, T. J., and Lister, D. H.: Updated high-resolution grids of monthly climatic observations - the CRU TS3.10 Dataset, Int. J. Climatol., 34, 623-642, https://doi.org/10.1002/joc.3711, 2014.
Hegerl, G. C., Zwiers, F. W., Allen, M. R., and Marengo J.: Detection of Climate Change and Attribution of Causes, in: Climate Change 2001: The Scientific Basis. Contribution of Working Group I to the Third Assessment Report of the Intergovernmental Panel on Climate Change, edited by: Houghton, J. T., Ding, Y., Griggs, D. J., Noguer, M., van der Linden, P. J., Dai, X., Maskell, K., and Johnson, C. A., Cambridge University Press, Cambridge, UK and New York, NY, USA, 881 pp., 2001.

IPCC: Managing the Risks of Extreme Events and Disasters to Advance Climate Change Adaptation. A Special Report of Working Groups I and II of the Intergovernmental Panel on Climate Change, edited by: Field, C. B., Barros, V., Stocker, T. F., Qin, D., Dokken, D. J., Ebi, K. L., Mastrandrea, M. D., Mach, K. J., Plattner, G.-K., Allen, S. K., Tignor, M., and Midgley, P. M., Cambridge University Press, Cambridge, UK, and New York, NY, USA, 582 pp., 2012.

IPCC: Climate Change 2013: The Physical Science Basis. Contribution of Working Group I to the Fifth Assessment Report of the Intergovernmental Panel on Climate Change, edited by: Stocker, T. F., Qin, D., Plattner, G.-K., Tignor, M., Allen, S. K., Boschung, J., Nauels, A., Xia, Y., Bex, V., and Midgley, P. M., Cambridge University Press, Cambridge, UK and New York, NY, USA, 1535 pp., 2013.

Knutti, R., Sedláček, J., Sanderson, B. M., Lorenz, R., Fischer, E. M., and Eyring V.: A climate model projection weighting scheme accounting for performance and interdependence, Geophys. Res. Lett., 44, 1909-1918, https://doi.org/10.1002/2016GL072012, 2017.

Legates, D. R. and Davis, R. E.: The continuing search for an anthropogenic climate change signal: limitations of correlation based approaches, Geophys. Res. Lett., 24, 2319-2322, 1997.

Mearns, L. O., Gutowski, W. J., Jones, R., Leung, L.-Y., McGinnis, S., Nunes, A. M. B., and Qian, Y.: A regional climate change assessment program for North America, Eos, Trans. Amer. Geophys. Union, 90, 311-312, 2009.

Murphy, A. H.: Skill scores based on the mean square error and their relationships to the correlation coefficient, Mon. Weather Rev., 116, 2417-2424, 1988.

Pierce, D. W., Barnett, T. P., Santer, B. D., and Gleckler, P. J.: Selecting global climate models for regional climate change studies, P. Natl. Acad. Sci. USA, 106, 8441-8446, https://doi.org/10.1073/pnas.0900094106, 2009.

Pincus, R., Batstone, C. P., Hofmann, R. J. P., Taylor, K. E., and Gleckler, P. J.: Evaluating the present-day simulation of clouds, precipitation, and radiation in climate models, J. Geophys. Res., 113, D14209, https://doi.org/10.1029/2007JD009334, 2008.

Radić, V. and Clarke, G. K. C.: Evaluation of IPCC models' performance in simulating late-twentieth-century climatologies and weather patterns over North America, J. Climate, 24, 5257-5274, 2011.

Schneider, U., Becker, A., Finger, P., Meyer-Christoffer, A., Rudolf, B., and Ziese, M.: GPCC Full Data Reanalysis Version 6.0 at $0.5^{\circ}$ : Monthly Land-Surface Precipitation from Rain-Gauges built on GTS-based and Historic Data, https://doi.org/10.5676/DWD_GPCC/FD_M_V7_050, 2011.

Schneider, U., Becker, A., Finger, P., Meyer-Christoffer, A., Ziese, M., and Rudolf, B.: GPCC's new land surface precipitation climatology based on quality-controlled in situ data and its role in 
quantifying the global water cycle, Theor. Appl. Climatol., 115, 15-40, https://doi.org/10.1007/s00704-013-0860-x, 2014.

Taylor, K. E.: Summarizing multiple aspects of model performance in a single diagram, J. Geophys. Res.-Atmos., 106, 7183-7192, 2001.

Taylor, K. E., Stouffer, R. J., and Meehl G. A.: An Overview of CMIP5 and the experiment design, B. Am. Meteorol. Soc., 93, 485-498, https://doi.org/10.1175/BAMS-D-11-00094.1, 2012.

van der Linden, P. and Mitchell, J. F. B. (Eds.): ENSEMBLES: Climate change and its impacts: Summary of research and results from the ENSEMBLES project. Met Office Hadley Centre, FitzRoy Road, Exeter, UK, 160 pp., 2009.
Willmott, C. J. and Matsuura, K.: Terrestrial Air Temperature and Precipitation: Monthly and Annual Time Series (1950-1999), available at: http://climate.geog.udel.edu/ climate/html_pages/ README.ghcn_ts2.html (last access: 16 October 2017), 2001.

$\mathrm{Xu}, \mathrm{Z}$., Hou, Z., Han, Y., and Guo, W.: A diagram for evaluating multiple aspects of model performance in simulating vector fields, Geosci. Model Dev., 9, 4365-4380, https://doi.org/10.5194/gmd-9-4365-2016, 2016. 